\title{
TOWARDS THE UNIFICATION OF QUANTUM DYNAMICS, RELATIVITY AND LIVING ORGANISMS
}

\author{
Arturo Tozzi \\ Center for Nonlinear Science, University of North Texas \\ 1155 Union Circle, \#311427Denton, TX 76203-5017 USA \\ Computational Intelligence Laboratory, University of Manitoba, Winnipeg, Canada \\ Winnipeg R3T 5V6 Manitoba \\ tozziarturo@libero.it \\ James F. Peters \\ Department of Electrical and Computer Engineering, University of Manitoba \\ 75A Chancellor's CircleWinnipeg, MB R3T 5V6 CANADA and \\ Department of Mathematics, Adıyaman University, 02040 Adıyaman, Turkey \\ James.Peters3@umanitoba.ca
}

John S. Torday (Corresponding Author)

Department of Pediatrics, Obstetrics and Gynecology

Evolutionary Medicine Program

David Geffen School of Medicine

University of California- Los Angeles

The unexploited unification of quantum physics, general relativity and biology is a keystone that paves the way towards a better understanding of the whole of Nature. Here we propose a mathematical approach that introduces the problem in terms of group theory. We build a cyclic groupoid (a nonempty set with a binary operation defined on it) that encompasses the three frameworks as subsets, representing two of their most dissimilar experimental results, i.e., 1) the commutativity detectable both in our macroscopic relativistic world and in biology; 2) and the noncommutativity detectable both in the microscopic quantum world and in biology. This approach leads to a mathematical framework useful in the investigation of the three apparently irreconcilable realms. Also, we show how cyclic groupoids encompassing quantum mechanics, relativity theory and biology might be equipped with dynamics that can be described by paths on the twisted cylinder of a Möbius strip.

KEYWORDS: Einstein; manifold; Hilbert space; Abelian; living cell; Borsuk-Ulam theorem; topology.

Both general relativity (GR) and quantum physics (QF) describe Nature using mathematical structures such as tensors and probability theory (Yilmaz, 1982; Comte, 1996; Fre, 2013). The unification of GR and QF stands for the Holy Grail in physics: the two issues, despite the fact that both work very well in the description of different features of Nature, seem to be incomparable and not reducible to a unified, self-consistent framework. Several efforts have been provided, in order to correlate them (e.g., Ghose, 1997; Skalsky, 2010; Fre, 2013; Elitzur, Dolev, Kolenda, 2017; Aerts, 2017), but they tend to paint a somewhat incomplete picture of the milieu described by GR and QF. Indeed, quantum dynamics and special relativity display very different behaviours and features, starting from the very subtending mathematical structures. General relativity is described on a 3+1-dimensional pseudo-Riemannian manifold with tensor fields obeying certain partial differential equations, while quantum field theory is described on an $\mathrm{R}^{4}$ projective Hilbert space, with operatorvalued fields obeying certain Lorentz-invariant partial differential equations and commutation relationships (Tegmark 2008). Therefore, in the Euclidean framework, describable by three spatial dimensions plus time, we use vectors equipped with just one basis, while in the projective Hilbert space of quantum mechanics, equipped with infinite dimensions, we make use of vectors with different bases standing for sets of equivalence classes. In other words, the macroscopic world of relativity has an orthonormal basis common to all the observables, i.e., a set of vectors $B$ in Euclidean or Hilbert space such that every vector can be written as a linear combination of vectors from $B$, while all vectors from $B$ have length 1 and any two of them are orthogonal (Tumulka, 2009).

On the other hand, the microscopic world of quantum dynamics displays different bases that are the source of the rather counterintuitive physical effects experienced during experimental observations. Efforts have been provided in order to correlate the two different manifolds. For example, techniques of deformation quantization, such as the Wigner-Weyl transform, have provided rules in order to strain the "classical" commutative algebra of observables into a quantum noncommutative one (Curtright et al., 2014). In this paper we will focus, rather than on manifolds, on another difference between the physical frameworks of GR and QF: commutability (Goodman, 2003). Indeed, in the macroscopic physical 
world dictated by GR, the binary operation of multiplication is commutative, i.e., changing the order of the operands does not change the result. This means that: $a b=b a$. In turn, in $Q F$ the linear operators representing a pair of physical variables do not commute, rather are mutually complementary. This means that $c d \neq d c$. Here we raise the question whether it is feasible to correlate the two mathematical operations of commutativity and noncommutativity in a unified scheme and to extend it to the biological realm too. Therefore, we ask here: is there a loop between commutativity and noncommutativity, which gives rise to a quantifiable graph? Has such hypothetical mathematical structure a physical meaning? Could its dynamics be described?

\section{LIVING BEINGS: COMMUTATIVE OR NONCOMMUTATIVE PROPERTIES?}

In the previous paragraph, we stated that noncommutativity can be found in the realm of quantum dynamics. Examples of complementary quantum properties which cannot all be observed or measured simultaneously are: position and momentum, energy and duration, spin on different axes, wave and particle, entanglement and coherence, and so on (Kalckar et al., 1996). Nevertheless, noncommutativity can be found also in another realm: the biological one. Indeed, biological issues are very intricate, because they lie somehow at the border between commutativity and noncommutativity. Living cells' dynamics might display mixed features: entropy, or negentropy (Schrodinger 2012), and chemiosmosis (Mitchell 1961) are non-commutative, whereas homeostasis is commutative. Furthermore, living matter, consisting of fermions, complies with both the principle of non-locality and the Pauli Exclusion Principle, because the first three quantum numbers, based on space, are non-commutative, whereas the fourth quantum number, based on time, is commutative (Morowitz 2004). "Life" is able to reconcile commutativity and noncommutativity because it originated as an ambiguity (Torday and Miller Jr, 2017). Indeed, according to some theories, the lipids contained in the snowball-like asteroids spontaneously form micelles when immersed in water (Shah, 1988; Deamer, 2017). Some scientists suggest that such micelles provided a protected space within a semi-permeable lipid membrane, which led to the generation of the First Principles of Physiology (Torday and Rehan, 2009), the warming and cooling of such micelles deforming and reforming faithfully on the Earth under the influence of the Sun. The faithful cycle of deformation-reformation is due to the hysteretic 'memory' of lipids, which are the pre-adaptations or exaptations that form the basis for the process of evolution (Gould and Vrba, 1982). In sum, quantum mechanics' noncommutativity and relativity's commutativity are both expressed by living beings' physiologic dynamics, such that an effort towards a full unification allows for also encompassing biology.

The peculiar standpoint of living beings. Reinforcing the sense of a cohesion and common origin between the physical and biologic, it is noteworthy that living beings' physiologic dynamics display intriguing relationships and homologies with QT and GR, not just relative to the issue of commutativity/non-commutativity. To make an example, the principle of non-locality is homologous with pleiotropy (Torday, 2018c) in the sense that the same gene is expressed in different physiologic traits. For example, epigenetic inheritance is homologous with the Brower fixed-point theorem from algebraic topology, the latter already used in order to describe quantum (Peters and Tozzi 2016) and biological issues (Tozzi et al., 2017; Tozzi et al., 2018). The theorem states, in plain terms, that it does not matter how much you slosh a cup of coffee: you will always find at least one drop of coffee in the same position as before: in biological terms, the unicellular state of the zygote acts as the 'point source' for the offspring (Torday and Miller, 2016a), generating the phenotype, which acts as the 'agent' for obtaining epigenetic 'marks' from the environment (Torday and Miller, 2016b). Bearing in mind that the developmental continuum from the zygote to the phenotype is a function of cell-cell interactions that comply with the First Principles of Physiology/Pauli Exclusion Principle, and that the phenotype as agent complies with topology, acting in compliance with epigenetic marks retained by the zygote, evolution as all of biology (Dobzhansky, 1973; (Torday, 2018a) integrates as a unity also the rather general algebraic topology.

The "Singularity of Nature" (Torday, 2018) would predict that biology complies with GR and QT. The following is a way to understand such interrelationships from the origins of life forward based on empiric evidence for epigenetic inheritance. There is experimental evidence for cells communicating independently of chemical or physical means (Scholkmann et al, 2013), offering a precedent for biologic cell-cell communication. In this sense the cell is acting as an observer of an everchanging environment, able to acquire epigenetic marks in order to accommodate such changes (Orias et al, 2017). The process of development recapitulates the history of the organism through cell-cell interactions (Torday, 2014), embryonic differentiation being due to the effects of soluble growth factors on pattern formation, target cells 'observing' the secretions of their neighboring cells of differing germ line origins (Wang et al, 1997). Over the course of such developmental cell-cell interactions, epigenetic marks originating from the egg and sperm (Gapp and Bohacek, 2018) are incorporated into somatic cells. "Observations" made by the adults are passed on to the offspring in order for them to attune themselves to environmental changes in advance of birth (Skinner, 2015).

The key to understanding the interrelationship between epigenetics and quantum theory lies in their common origins in homeostasis. It has been proposed that the cell and the atom are homologous, the electron and proton of a hydrogen atom in balance with one another as a 'template' for the First Principles of Physiology of the cell. When physiologic stress occurs, it generates Radical Oxygen Species (ROS), known to cause genetic mutations and duplications (Storr et al., 2013); under the constraints of the First Principles of Physiology, the cellular structure will remodel itself until it re- 
establishes its homeostatic set-point through what is otherwise referred to as evolution (Torday, 2015). To provide an example, the alveolus of the lung has remodeled itself over the course of evolution to optimize the ratio of the gasexchange surface area-to-blood volume, mediated by cell-cell interactions that determine the production of lung surfactant in compliance with the Law of Laplace (Bernhard, 2016). This process is under the homeostatic control of Parathyroid Hormone-related Protein (PTHrP), a stretch-sensitive gene (Torday et al., 1998). Similarly, the glomerulus has remodeled the fish kidney glomus through cell-cell interactions to optimize the physiologic levels of water and electrolytes on land (Smith 1953), which is also under the egis of PTHrP (Bosch et al., 1999). Both of these evolved traits are epigenetic in the sense that they are due to environmental effects on the cell, not to DNA mutations. As mentioned above, ROS can cause gene duplications such as those that occurred during the water-land transition (Romer 1949) for the PTHrP Receptor, Glucocorticoid Receptor (GR) and beta-Adrenergic Receptor (beta AR), all three of which are necessary for adaptation to land. To test this hypothesis, Xenopus laevis tadpole lungs were treated with PTHrP, which accelerated their lung structural and functional development (Torday et al., 2009), evidencing the role of lung PTHrP 'amplification' in the transition from water to land. Similar experiments have been done with GR (Shewade et al., 2017) and beta AR (Mori et al., 2013) ligands, evidencing their roles in vertebrate evolution in adapation to land.

Summarizing, apart from noncommutativity and commutativity, living beings also display other close relationships with both QR and GR, and this claim points once again towards the feasibility of a general scheme able to unify the three realms.

\section{PREVIOUS EFFORTS TOWARDS UNIFICATION}

Several efforts have been made in order to unify GR and QT, but results are missing. Many investigations aimed to provide transformation rules between the two manifolds of GR and QT. To make an example, Feffman et al. (2015) assessed the geometric Whitney problem on how a Riemannian manifold can be constructed to approximate a metric space. Deformation quantization (such as the above-mentioned Wigner-Weyl transform) is able to achieve a complete phase space formulation of quantum mechanics, equivalent to the Hilbert-space operator representation, with starmultiplications that are isomorphically parallel operator multiplications (Curtright et al., 2014). By expressing quantum mechanics in the same phase space as that of classical mechanics, the map achieved through the Wigner-Weyl transform facilitates recognition of quantum mechanics as a deformation of the classical one. However, such techniques are limited to the description of just a mere representation change from Hilbert space to phase space, because they are not able to build a successful quantization scheme, namely a method to produce a quantum theory out of a classical one. Furthermore, recent experimental efforts point towards the possibility of simultaneous observation of quantization and interference patterns (Piazza et al., 2015). However, Piazza et al.'s experiment stands for a technical device, rather than a proper conceptual framework able to unify GR and QT.

It is worth mentioning that special relativity has been tackled also by a noncommutative standpoint (Calmet and Fritz, 2015; Besnard and Bizi, 2017). Girelli and Livine (2004) argued that Deformed Special Relativity is obtained by imposing a maximal energy to Special Relativity and by deforming the Poincare symmetry in order to accommodate this requirement. Variations of the same procedure lead to a noncommutative space structure that preserves conservation laws. In turn, the Very Special Relativity's (VSR) approach demonstrated that that the subgroup of the Poincare group is sufficient to describe the spacetime symmetries of observed physical phenomena. Indeed, Das et al. (2011) introduced a novel noncommutative spacetime structure that enjoys the symmetries of deformed VSR: this trick allowed them to build a point particle Lagrangian that lives in a noncommutative phase space. Some Authors view QT as a deformation of the combinatorial or Hamiltonian quantization of three-dimensional gravity in the Chern-Simons formulation. In this approach, quantum groups replace the local isometry groups, and noncommutative spacetimes replace the classical model spacetimes (Schroers, 2011). Furthermore, Kalau and Malze (1995) derived an action for gravity in the framework of noncommutative geometry by using the Wodzicki residue. They achieved a gravity action for commutative geometry which is the usual Einstein-Hilbert action. They also provided a noncommutative extension given by the tensor product of the algebra of smooth functions on a manifold and a finite dimensional matrix algebra.

In contrast with the above-mentioned approaches, in the next paragraph we will provide a purely mathematical framework based on set theory, where the unification of the symplectic phase space of the macroscopic relativistic world, the noncommutative phase space of the microscopic quantum world and biological entities it is not strictly required.

\section{A CYCLIC GROUPOID ENCOMPASSES LOOPS BETWEEN COMMUTATIVE AND NONCOMMUTATIVE OPERATIONS}

Our goal, i.e., the achievement of a set that encompasses both the commutativity and noncommutativity subsets, cannot be tackled through the otherwise successful Fraenkel-Zermelo set, because some of its axioms cannot work properly in this peculiar context. Therefore, we start from a groupoid A, equipped with a commutative binary operation, and a groupoid $\mathrm{B}$, equipped with a noncommutative binary one. We require that a single observable must be either macroscopic (commutative, subjected to relativity and to biology) or microscopic (noncommutative, subjected to quantum dynamics 
and to biology). Here we show that a set $\mathrm{C}$ does exist, able to encompass both $\mathrm{A}$ and $\mathrm{B}$. The procedure is illustrated in Figure 1.

The set $\mathrm{A}=\{\mathrm{a}, \mathrm{b}, \mathrm{c}\}$, while the operation $<_{0}>: \mathrm{AxA} \rightarrow \mathrm{A}$.

$\mathrm{A}<\mathrm{O}>\mathrm{b}=\mathrm{b}<\mathrm{0}>\mathrm{a}=\mathrm{b}$ reads: "( $\mathrm{a}, \mathrm{b})$ maps to $\mathrm{b}$ ", and "(b,a) maps to b".

In general, $\mathrm{x}<_{0}>\mathrm{y}=\mathrm{y}<_{0}>\mathrm{X}$ for all elements $\mathrm{x}, \mathrm{y}$ in $\mathrm{A}$. This means that $\left(\mathrm{A},<_{0}>\right)$ is an Abelian groupoid, and the operation o is commutative.

The set $\mathrm{B}=\{\mathrm{d}, \mathrm{e}, \mathrm{f}, \mathrm{g}\}$, while the operation $<_{\mathrm{o}}>$ : BxB $\rightarrow \mathrm{B}$.

$\mathrm{d}<_{0}>\mathrm{e}=\mathrm{e}$ reads: "( $\left.\mathrm{d}, \mathrm{e}\right)$ maps to e", and " $(\mathrm{e}, \mathrm{d})$ maps to e". Further, e $<_{\mathrm{o}}>\mathrm{f}=\mathrm{f}$ reads: "(e,f) maps to f", but $\mathrm{f}<\mathrm{o}>\mathrm{e}=\mathrm{f}$ does not map to $\mathrm{f}$ ".

This means that $\left(\mathrm{B},<_{0}>\right)$ is a non-Abelian groupoid, and the operation $<_{0}>$ is notcommutative.

Sets $\mathrm{A}$ and $\mathrm{B}$ are contained in $\mathrm{C}$. The set $\mathrm{C}=\{\mathrm{a}, \mathrm{b}, \mathrm{c}, \mathrm{d}, \mathrm{e}, \mathrm{f}, \mathrm{g}\}$, while the operation $[<\mathrm{o}>]: \mathrm{CxC} \rightarrow \mathrm{C}$.

$\mathrm{a}[<\mathrm{o}>] \mathrm{b}=\mathrm{b}[<\mathrm{0}>] \mathrm{a}=\mathrm{b}$ commutes.

$\mathrm{a}[<\mathrm{0}>] \mathrm{d}=\mathrm{d}[<\mathrm{0}>] \mathrm{a}=\mathrm{d}$ commutes.

$\mathrm{b}[<\mathrm{O}>] \mathrm{e}=\mathrm{e}[<\mathrm{O}>] \mathrm{b}=\mathrm{e}$ commutes.

$\mathrm{c}\left[<_{0}>\right] \mathrm{f}=\mathrm{f}[<\mathrm{O}>] \mathrm{c}=\mathrm{f}$ commutes.

$\mathrm{e}\left[<_{\mathrm{o}}>\mathrm{f}=\mathrm{f}, \mathrm{f}[<\mathrm{O}>] \mathrm{e}=\mathrm{e}\right.$ does not commute, and so on.

Therefore, $\left(\mathrm{C},\left[<_{0}>\right]\right)$ is a non-Abelian groupoid, and the operation $\left[<_{0}>\right]$ is not commutative. 


\section{Set A}

Set B

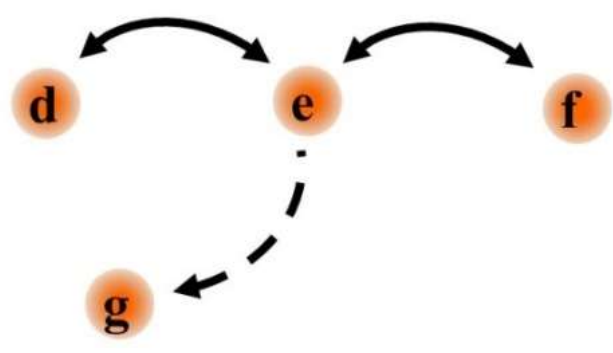

\section{Set C}

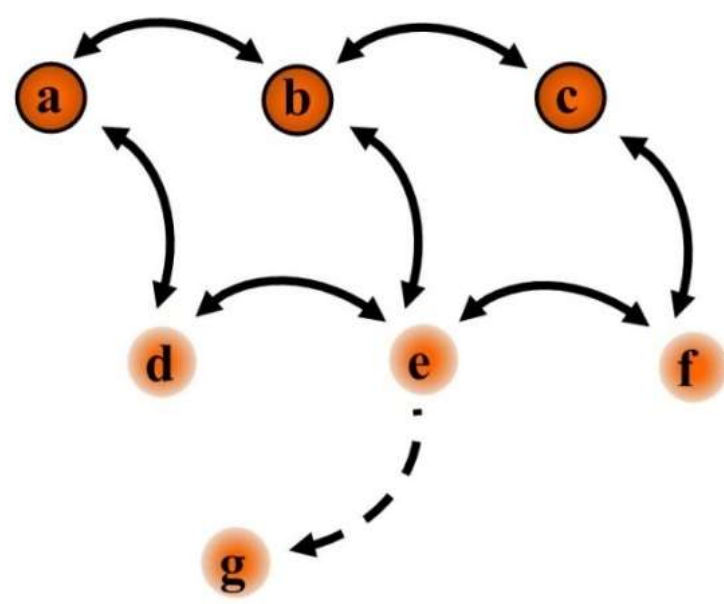

Figure 1. Three sets in terms of cyclic groupoids. The solid, double-arrowed lines stand for commutative relationships, while the dotted arrows for noncommutative ones. The circles $\mathrm{a}, \mathrm{b}$ and $\mathrm{c}$ (encompassed in the set A) stand for physical observables experimentally detectable in our macroscopic relativistic or biological world, equipped with commutative binary operation properties. The circles $\mathrm{d}, \mathrm{e}, \mathrm{f}$ and $\mathrm{g}$ (encompassed in the set $\mathrm{B}$ ) stand for physical observables experimentally detectable in our microscopic quantum world and in the biological realm, equipped with noncommutative binary operation properties. The set $\mathrm{C}$ embeds both the sets $\mathrm{A}$ and $\mathrm{B}$.

\section{A MATHEMATICAL UNIFICATION VIA CYCLIC GROUPOIDS AND SPACETIME FUSION CATEGORIES}

This section briefly introduces the unification of Abelian and non-Abelian cyclic groupoids in a fusion category. Fusion categories are introduced in Henriques and Penneys (2016) using the Connes fusion operator $\bigotimes$ (Connes, 1994; Bartels, A., Douglas, C.L., Henriques, A. 2014), elaborated in Wassermann (1998). 
Let $A_{\circ} \equiv(A, \circ), B_{\circ} \equiv(B, \circ)$ denote a pair of cyclic groupoids, each with a binary operation o.

Let $[x]$ denote a Connes fusion operator, which is an associative product on bimodules (in our case, a bimodule is the Cartesian product of a pair of cyclic groups) and let $g: A_{\circ} \times B_{\circ} \rightarrow A_{\circ}[\times] B_{\circ}$ be an isomorphishm on a pair of cyclic groupoids onto the fusion of the groupoids $A_{\circ}, B_{\circ}$. The fusion of these groupoids is represented in Figure 2. Putting this together, we obtain

$$
\left(A_{\circ}, B_{\circ}\right) \stackrel{g}{\mapsto} A_{\circ}[\times] B_{\circ} \mapsto A_{\circ} \cup B_{\circ}=(A \cup B, \circ)
$$

Thanks to the Connes fusion operator, we achieve a mathematical framework in the form of a fusion category, which is useful in reasoning about the unification of quantum theory, relativity and biology. A fusion category consists of a collection of objects and a collection of morphisms (maps between objects in a fusion category). The focus here is on fusion categories in which the objects are cyclic groupoids and associative products (fused) bimodules, while the morphisms are mappings on pairs of cyclic groupoids or fused cyclic groupoids.

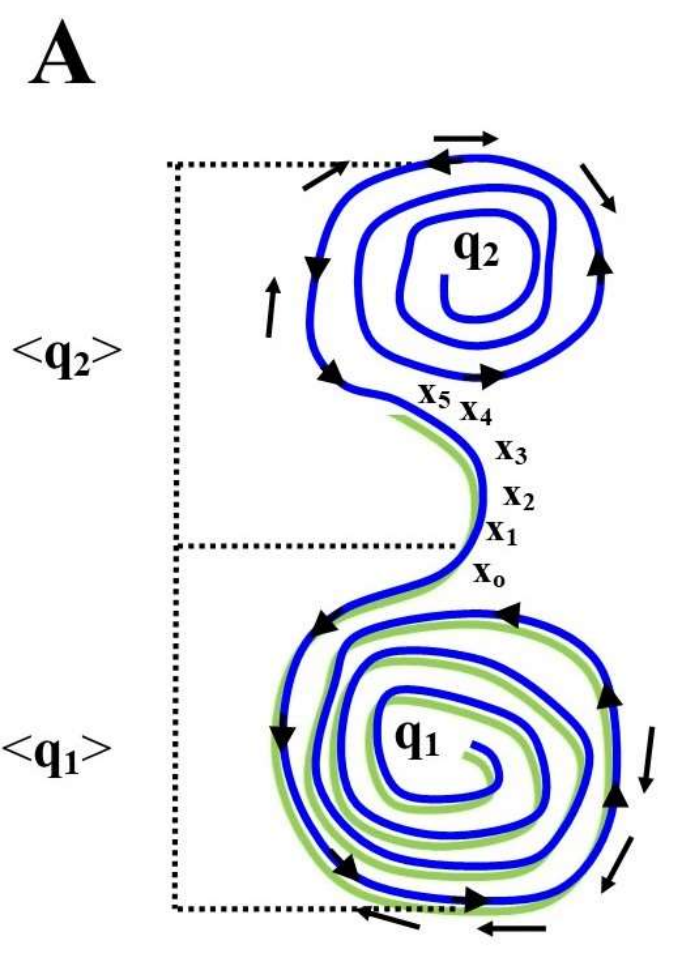

\section{B}

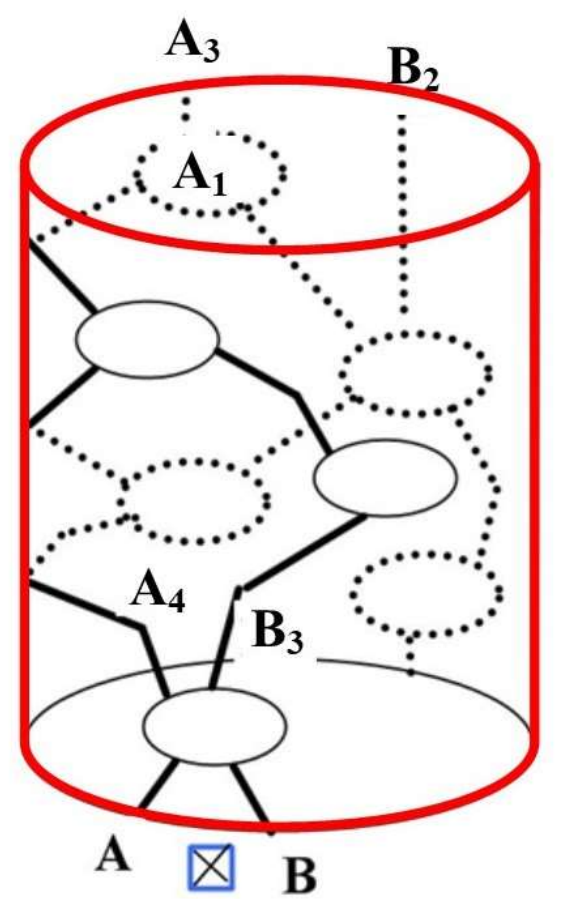

Figure 2A: Generators for a Cyclic Groupoid.The cyclic groupoid $A_{\circ}$ has a generator $\left\langle q_{1}\right\rangle$. Similarly, the cyclic groupoid $B_{\circ}$ has a generator $\left\langle q_{2}\right\rangle$. This means, for example, each element $A_{i}=q_{1} \circ 2 q_{1} \cdots \circ k q_{1}$ in $A_{\circ}$ is a linear combination of the generator $q_{1}$. The fusion of the two groupoids results in a new groupoid, namely, $A_{\circ} \cup B_{\circ}=(A \cup B, \circ)$.

Figure 2B: Spacetime View of a Fusion Category. A spacetime view of a fusion category is shown in Fig. X(b).

Over time,

$A_{1} \otimes A_{1} \mapsto A_{1} \otimes A_{2} \mapsto A_{1} \otimes A_{2} \otimes A_{3} \mapsto A_{1} \otimes A_{2} \otimes A_{3} \otimes A_{4} \equiv A$

and

$B_{1} \otimes B_{1} \mapsto B_{1} \otimes B_{2} \mapsto B_{1} \otimes B_{2} \otimes B_{3} \equiv B$

which are Connes fused, resulting in $A[\times] B$, that is an object in the spacetime fusion category. 


\section{PHYSICAL MEANING OF A SET ENCOMPASSING QUANTUM MECHANICS, RELATIVITY THEORY AND BIOLOGY}

We would like to posit a speculative claim, which is a theoretical effort to try to answer to the crucial question: what is the physical meaning of the set $\mathrm{C}$ we described, i.e., the set which covers quantum issues, relativity and living beings as a unitary accounting? As suggested by the Nobel Prize t'Hooft (1999), a theory can be developed that does not postulate the quantum states as being the central cosmic starting point, but rather as a classical deterministic background. In this account, quantum mechanics, relativity and biology, with their burden of space, time, matter, and causality, turn out to be concepts derived from a deeper layer of reality (Bohm, 1982?), where space-time could be built with quantum entanglement (Van Raamsdonk 2010; Hogan and Kwon, 2017). The candidate to a completely unified theory could be the continuum limit of a classical, deterministic discrete theory, at a string level where information loss is allowed. Our set $\mathrm{C}$ might lie at an ultramicroscopic level, closed to the Planck length, which, after information loss, might give rise to equivalence classes, i.e., the discrete sets which stand for quantum mechanics, relativity and biology. Such hypothetical unifying manifold must display rather general dynamics, in order to encompass the whole of subsets described by QT, GR and biology. Therefore, the distinction among subsets must disappear: our conventional (physical and biological) representations of identity, individual, relative observers, causality, cellular self-organization, closed Jordan curves separating an internal and an external environment, have to vanish. To make an example, some Authors stressed the central role of cell-cell communication for development, homeostasis and regeneration/repair, which is predicated upon one cell-type signaling to another as a representation of Einstein's 'observer effect' in GR (Einstein, 1916). This way of thinking about cell-cell communication transcends the present, referencing earlier and earlier iterations of the organism, all the way back to its unicellular origin (Torday 2018a). Beyond that, based on evolution as pre-adaptive, and biology as self-referentially self-organized, the unicell references the Singularity that existed before the Big Bang (Torday, 2018a). In particular, in order to describe the unity subtending quantum, relativistic and biological issues, our unifying set must be void of the concept of internal and external, which stand just for a human effort to restrain and confine quantifiable observables. We will focus here on the dynamics that must be displayed by this unifying, general manifold void of internal/external borders. In doing so, we need at first to introduce the Borsuk-Ulam theorem (BUT).

Describing the world: the Borsuk-Ulam theorem. Results from far-flung scientific disciplines point towards the BUT as a universal principle able to describe and quantitatively assess otherwise elusive quantum, physical and biological activities, such as logistic maps of chaotic activities (Tozzi and Peters, 2016b), (spatial) fractals and (temporal) power laws (Tozzi et al., 2017a), symmetry breaks (Tozzi and Peters, 2016b), nervous multidimensional dynamics (Peters et al., 2017a), neural thermodynamic activity and energy requirements/constraints during both spontaneous and evoked nervous function (Tozzi and Peters, 2017b).

The BUT suggests that the properties endowed in physical and biological spaces can be translated to abstract mathematical ones (Tozzi and Peters, 2016a). In plain words, the BUT states that antipodal points in $n+1$ dimensional Euclidean space map to identical function values in n-dimensional Euclidean space. In our case, $n+1$ becomes $3+1$ in spacetime and $n$ becomes conventional Newtonian (observer) space. In more technical terms, antipodal points embedded in a higher dimension space map to matching descriptions in a lower dimensional space, provided that the mapping is continuous (Dodson and Parker, 1997; Matoušek 2003). The original formulation of BUT displays versatile ingredients which can be modified, resulting in useful extensions of its rather simple scenario (Tozzi et al., 2017a). For example, antipodal points can be replaced by antipodal regions, or shapes, with matching description (Tozzi and Peters, 2016b). Instead of points, novel BUT variants allow the assessment (from one dimension to another) of trajectories, functions, vectors and tensors, particle trajectories in phase spaces, information and activities such as entropies (Tozzi and Peters, 2017c). Also, the two features do not need to be perfectly antipodal: the only requirement is that they must not share points in common, being fully separated in the higher-dimensional manifold (Tozzi et al., 2017a). BUT variants hold not just for concave structures, such as the circumferences and spheres described by the classical BUT, but also for flat and concave structures (Tozzi 2016), such as the rather intricate trajectories detected in several systems' dynamics (Sengupta et al., 2016). Furthermore, the dimensions described by BUT do not stand just for spatial dimensions (such as a circle and a sphere), but also for abstract dimensions (such as for example, fractal measurements and time intervals) (Tozzi and Peters, 2016b).

Erasing borders: Möbius strips come into play. The BUT copes with projections and mappings among different functional dimensions, while physical and biological dynamics are experimentally described not in such terms of mappings and projections, rather of paths and trajectories taking place in phase spaces. In order to tackle this issue, we are allowed to transport the scenario described by BUT to a peculiar phase space, i.e., a Möbius strip, so that antipodal points can be assessed in terms of trajectories taking place on a rather simple abstract manifold. The Möbius strip, also called the twisted cylinder, is a one-side surface which, when is embedded in three-dimensional Euclidean space, displays just one boundary (Möbius, 1858; Starostin and van der Heijden 2007; t'Hooft 2018),. A Möbius strip can be built by taking a paper strip and giving it a half-twist, then joining the ends in order to form a loop. This means that a line that starts from the seam down the middle meets back at the seam, but at the other side. If continued, the line meets the starting point, in a point that is double the length of the original strip. This single continuous curve may be described either through a parameterized subset of a three-dimensional Euclidean space, or through cylindrical polar coordinates. 
Topologically, the Möbius strip can be defined as the square $[0,1] \times[0,1]$, with its top and bottom sides identified by the relation $(\mathrm{x}, 0) \sim(1-\mathrm{x}, 1)$ for $0 \leq \mathrm{x} \leq 1$.

The transport of the BUT's antipodal points to the one-side surface of such twisted cylinder may be technically achieved. Indeed, if we embed the trajectories of two BUT matching functions $\mathrm{x}$ and $-\mathrm{x}$ (Figure 3A) on a Möbius strip, we achieve a closed, continuous loop where the two functions are allowed to travel along constrained trajectories. It is easy to see that a piece of strip of a given length, standing for a time interval, may display both $\mathrm{x}$ and $-\mathrm{x}$ at the same time (Figure 3B). The BUT dictates are preserved because, even if the two matching features are simultaneous, they do not have points in common: indeed, they lie on the opposite surface of the same strip. To make an example in nervous dynamics' terms (Watanabe et al., 2014; Ezaki et al., 2017), this means that the oscillations' trajectories of two areas which activate together can be followed in subsequent times, even when their matching activation has disappeared (Figure 3C).

In the last paragraph, we will describe why and how a Möbius strip perfectly fits the requirement for our unifying manifold.

A

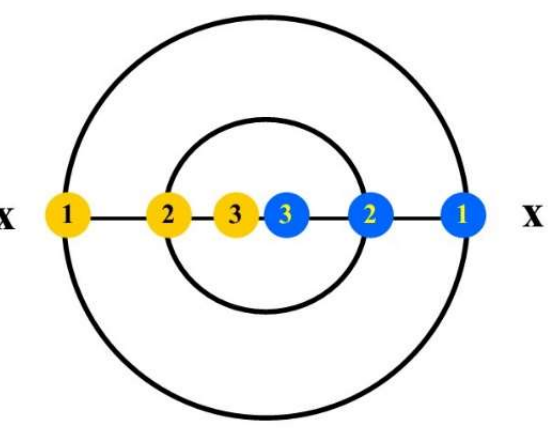

C

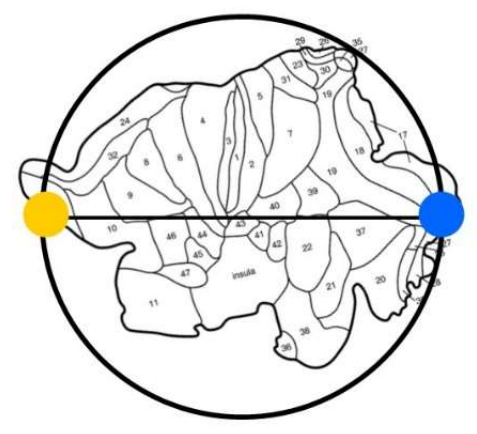

B
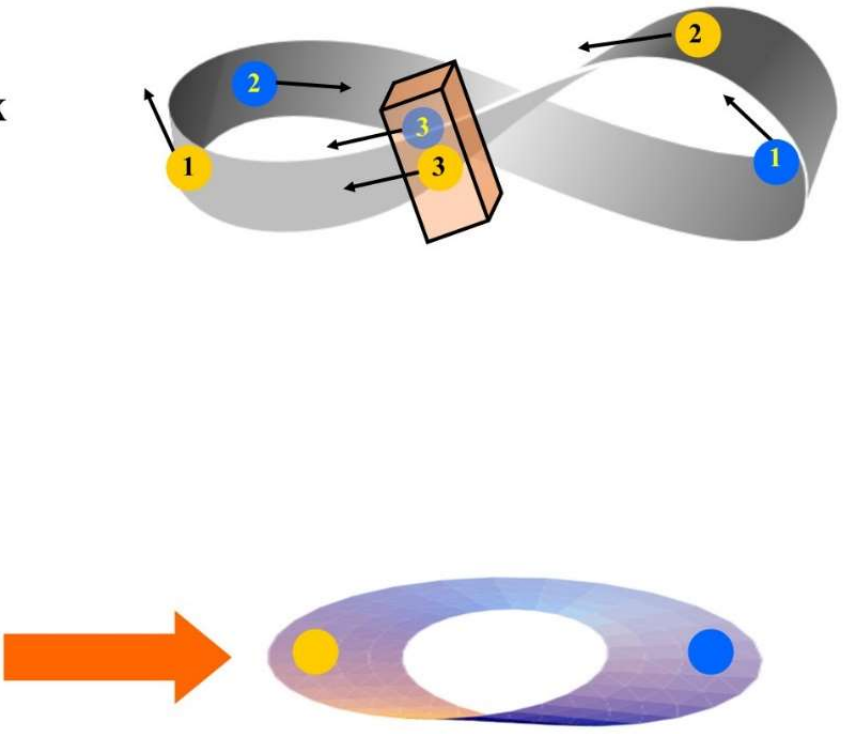

Figure 3. Transport of the BUT theorem on a Möbius strip. Figure 3A. Changing the radius of the hypersphere makes the antipodal points more or less close. Close to the center, the two points (marked with the number 3) are almost superimposed. Figure 3B. The movements of the antipodal points can be described in terms of trajectories on a Möbius strip. The parallelepiped stands for a slice of time, where both the antipodal features occur simultaneously. Figure 3C. A theoretical example from neuroscience is provided. A cerebral hemisphere is unfolded and flattened into a twodimensional reconstruction (Van Essen, 2005) that can be embedded into a circular manifold. When two antipodal areas display simultaneously a feature in common, e.g., the same value of pairwise entropy (Watanabe et al. 2014), we achieve a topological description assessable in terms of BUT (left side). Such two areas and their subsequent dynamics can be easily visualized and assessed in terms of trajectories taking place on an abstract twisted cylinder (right side). 


\section{CONCLUSIONS}

We propose a mathematical approach which allows to join, in a single group theoretic framework, both the commutative and noncommutative operations that stand for the macroscopic relativistic world, the microscopic quantum realm, and living entities. Our framework paves the way to a fully mathematical approach which unifies quantum dynamics, general relativity and biology. Another possibility, not explored here, would be to use an involutive function between the opposite categories of commutativity and noncommutativity, in order to attain a dagger structure with the adjoining of a morphism being the inverse. This treatment would make it possible to achieve morphisms that are unitary in finite dimensional Hilbert spaces, in order to build a dagger symmetric monoidal category (Blute and Panangaden, 2011), which is also compactly or and? closed, resulting in completeness results that extend the use of Hilbert spaces to relativity issues. To accomplish a dagger category, a fusion category is equipped with a dagger $\uparrow$ so that morphisms $f: A \rightarrow B$ have a twoside inverse (Huenen and Karvoven, 2016).

Once having been achieved, a unifying set which encompasses quantum, physical and biological dynamics, we looked for the possible physical meaning of this rather general mathematical structure. Starting from the BUT, which stands for a general principle able to describe a large amount of physical and biological dynamics, we showed that antipodal features with matching descriptions (say, two far apart brain areas that are activated simultaneously and display the same value of entropy) can be described in terms of closed paths on a Möbius strip. With BUT, we are facing a framework based just on mappings and projections among different activity levels: once established, the validity of the theorem in the assessment of physical and biological activities requires an affordable procedure in order to visualize and process the hypothetical set encompassing them. Here we have shown how the features described by BUT that occur on an orientable manifold with positive-curvature can be described in terms of paths on a non-orientable manifold, i.e., a Möbius strip. Indeed, the Möbius strip has the mathematical property of being unorientable, and this allows to elucidate some physical and biological puzzling functions. At first, the description of phase space trajectories, detected through experimental observation, on a twisted cylinder instead of on the classical three-dimensional-plus time phase space, allows to notice possible unexpected correlations. The possibility to locate oscillations on a Möbius strip allows the assessment of simultaneous activities that are spatially separated. This allows, for example, the evaluation in a single framework of the quantum entanglement, or the simultaneous activation of the primary sensitive cortex and the frontal areas during a visualrelated task. Furthermore, the non-orientability of the Möbius strip which stand for our unifying manifold does not allow us to separate anymore an external from an internal: this fists with our goal to find a rather general feature able to encompass QT, GR and biology. We become able to evaluate quantum, physical and biological dynamics in terms of trajectories taking place onto the well-established, easily manageable phase space of a twisted cylinder. Indeed, the BUT requirements (i.e., two features with no points in common and the proper mappings) are fully preserved when mapped to a Möbius strip. The transport of the BUT apparatus to a Möbius strip displays also another invaluable advantage: because the mapping achieved by making a trip around a twisted cylinder is an inversion, this permits the preservation of the invariance under inversions, therefore obeying to the laws of conservation of energy and information. In conclusion, the study of cosmic, physical and biological oscillations on a twisted cylinder (instead of three-dimensional Euclidean phase spaces, or of Hilbert multidimensional manifolds) is justified by the BUT framework and might pave the way to the detection and assessment of unexpected relationships among dissimilar quantum, relativistic and biological activities.

\section{REFERENCES}

1) Aerts, D.; Aerts, S. 2017. Towards a general operational and realistic framework for Quantum mechanics and Relativity Theory, in A. Elitzur, S. Dolev, N. Kolenda, Eds., Quo Vadis Quantum Mechanics, Springer, 2017.

2) Barlow DJ, Hollinshead CM, Harvey RD, Kudsiova L, Lawrence MJ. Memory effects of monolayers and vesicles formed by the non-ionic surfactant, 2C(18)E(12). J Colloid Interface Sci. 2007 Dec 15;316(2):74150.

3) Bartels, A., Douglas, C.L., Henriques, A. 2014. Dualizability and index of subfactors. arXiv 1110.5671v2,1-36.

4) Bernhard W. Lung surfactant: Function and composition in the context of development and respiratory physiology. Ann Anat. 2016 Nov;208:146-150.

5) Besnard F, Bizi N. 2017. On the definition of spacetimes in Noncommutative Geometry: Part I. arXiv:1611.07830v3 [math.OA] 13 Mar 2017

6) Blute R., Panangaden P. 2011. Dagger categories and formal distributions. Lecture Notes in Physics 813, Springer, Heidelberg, 421-436, DOI 10.1007/978-3-642-12821-9_6, MR2767050.

7) Bohm D. Wholeness and the Implicate Order. Routledge, New York, 2002.

8) Borsuk K. 1933. Drei Sätzeüber die n-dimensionale euklidische Sphäre. FundamentaMathematicae. 20: 177-190.

9) Borsuk K. 1969. Fundamental retracts and extensions of fundamental sequences, 64, no. 1, 55-85. 
10) Bosch RJ, Rodríguez-Puyol D, Bover J, Rodríguez-Puyol M. Parathyroid Hormone-related protein: roles in the glomerulus. Exp Nephrol. 1999 May-Jun;7(3):212-6.

11) Calmet X, Fritz C. 2015. Inflation on a Non-Commutative Space-Time. arXiv:1506.04049v1 [hep-th] 12 Jun 2015

12) Connes, A. 1994. Noncommutative Geometry. Academic Press Inc., San Diego, CA.

13) Curtright, T. L.;Fairlie, D. B.; Zachos, C. K. 2014. A Concise Treatise on Quantum Mechanics in Phase Space. World Scientific. ISBN 9789814520430.

14) Comte C. 1996. Symmetry, relativity and quantum mechanics, Il Nuovo Cimento, vol. 111 B, no. 8, 937 956.

15) Das S, Ghosh S, Mignemi S. 2011. Non-commutative spacetime in very special relativity. Physics Letters A. Volume 375, Issue 37, 29 August 2011, Pages 3237-3242. https://doi.org/10.1016/j.physleta.2011.07.024.

16) Deamer D. The Role of Lipid Membranes in Life's Origin. Life (Basel). 2017 Jan, 17;7(1).

17) Dobzhansky T. 1973. Nothing in Biology Makes Sense Except in the Light of Evolution. American Biology Teacher 35: 125-129.

18) Dodson CTJ, Parker PE. 1997. A user's guide to algebraic topology, Kluwer, Dordrecht, Netherlands, 1997, xii+405 pp. ISBN: 0-7923-4292-5, MR1430097.

19) Einstein A. 1916. Relativity: The Special and General Theory. Holt and Company, New York.

20) Elitzur, A.; Dolev, S.; N. Kolenda, Eds. 2017. Quo Vadis Quantum Mechanics, Springer.

21) Ezaki T, Watanabe T, Ohzeki M, Masuda N. 2017. Energy landscape analysis of neuroimaging data. Philos Trans A Math Phys Eng Sci. 2017 Jun 28;375(2096). pii: 20160287. doi: 10.1098/rsta.2016.0287.

22) Fefferman C, Ivanov S, Kurylev Y, Lassas M, Narayanan H. 2015. Reconstruction and interpolation of manifolds I: The geometric Whitney problem. arXiv:1508.00674

23) Fré, P.G. 2013. Gravity, a Geometrical Course. Vol. 1: Development of the Theory and Basic Physical Applications, Springer, DOI 10.1007/978-94-007-5361-7

24) Gapp K, Bohacek J. Epigenetic germline inheritance in mammals: looking to the past to understand the future. Genes Brain Behav. 2018 Mar;17(3):e12407.

25) Ghose, P. 1997. Violatioj of signal locality and unitarity in a merger of quantum mechanics and general relativity, Pramana 49, no. 1, 65-69.

26) Girelli F, Livine ER. 2004. Special relativity as a non commutative geometry: Lessons for deformed special relativity. Phys.Rev. D81 (2010) 085041. DOI: 10.1103/PhysRevD.81.085041

27) Goodman F.2003. Algebra: Abstract and Concrete, Stressing Symmetry, 2e. Prentice Hall. ISBN 0-13067342-0.

28) Gould, Stephen Jay; Vrba, Elizabeth S. (1982). Exaptation - a missing term in the science ofform. Paleobiology 8 (1): 4-15.

29) Henriques, A. and Penneys, D. 2016. Bicommutant categories from fusion categories. arXiv 1511.05226v2,1-35.

30) Heuen, C., Karvoven, M. 2016. Monads on dagger categories. Theory and Applications of Categories, 31 , no. 35, 1016-1043, MR3584697.

31) Hogan C, Kwon O. 2017. Models of Exotic Interferometer Cross-Correlations in Emergent Space-Time. arXiv: 1711.05514v4 [gr-qc] 17 Dec 2017

32) Kalau W, Malze M. 1995. Gravity, non-commutative geometry and the Wodzicki residue.Journal of Geometry and Physics, Volume 16, Issue 4, July 1995, Pages 327-344. https://doi.org/10.1016/03930440(94)00032

33) Kalckar J, Bohr N, Rosenfeld L, Rüdinger E, AaserudF. 1996. Foundations of Quantum Physics II (19331958). Elsevier. p. 210. ISBN 978-0-444-89892-0. Retrieved 2011-10-24.

34) Matoušek, J. 2003. Using the Borsuk-Ulam Theorem. Lectures on topological methods in combinatorics and geometry. Written in cooperation with Anders Björner and Günter M. Ziegler.Springer-Verlag, Berlin, 2003. xii+196 pp. ISBN: 3-540-00362-2.

35) Mitchell P. Coupling of phosphorylation to electron and hydrogen transfer by a chemi-osmotic type of mechanism". Nature 191: 144-148, 1961

36) Möbius AF. 1858. Werke, Vol. 2. p. 519.

37) Mori S, Moriyama Y, Yoshikawa K, Furukawa T, Kuroda H. $\beta$ Adrenergic signaling promotes posteriorization in Xenopus early development.Dev Growth Differ. 2013 Apr;55(3):350-8.

38) Morowitz H. The Emergence of Everything.Oxford University Press, Oxford, 2004.

39) Orias E, Singh DP, Meyer E. Genetics and Epigenetics of Mating Type Determination in Paramecium and Tetrahymena. Annu Rev Microbiol. 2017 Sep 8;71:133-156.

40) Peters JF, Tozzi A. 2016. Quantum Entanglement on a Hypersphere. Int J Theoret Phys, 1-8. doi:10.1007/s10773-016-2998-7. 
41) Peters JF, Ramanna S, Tozzi A, İnan E. 2017a. Bold-Independent Computational Entropy Assesses Functional Donut-Like Structures in Brain fMRI Images. Front Hum Neurosci. 2017 Feb 1;11:38. doi: 10.3389/fnhum.2017.00038.

42) Piazza L, Lummen TT, Quiñonez E, Murooka Y, Reed BW, Barwick B, Carbone F. 2015. Simultaneous observation of the quantization and the interference pattern of a plasmonic near-field. Nat Commun. 2015 Mar 2;6:6407. doi: 10.1038/ncomms7407.

43) Romer, A.S. 1949. The Vertebrate Story. University of Chicago Press, Chicago.

44) Schrodinger E. What is Life? Cambridge University Press, Cambridge, 2012.

45) Schroers BJ. 2011. Quantum gravity and non-commutative space-times in three dimensions: a unified approach. Acta PhysicaPolonica B Proceedings. Vol. 4 (2011) Supplement No 3

46) Sengupta B, Tozzi A, Coray GK, Douglas PK, Friston KJ. 2016. Towards a Neuronal Gauge Theory. PLOS Biology 14 (3): e1002400. doi:10.1371/journal.pbio.1002400.

47) Scholkmann F, Fels D, Cifra M. Non-chemical and non-contact cell-to-cell communication: a short review. Am J Transl Res. 2013 Sep 25;5(6):586-93.

48) Shah DO. Micelles: Microemulsions, and Monolayers: Science and Technology. CRC Press, Abingdon, 1998.

49) Shewade LH, Schneider KA, Brown AC, Buchholz DR. In-vivo regulation of Krüppel-like factor 9 by corticosteroids and their receptors across tissues in tadpoles of Xenopus tropicalis. Gen Comp Endocrinol. 2017 Jul 1;248:79-86.

50) Skalsky, V. 2010. The model of a flat (Euclidean) expansive homogeneous and isotropic relativistic universe in the light of the general relativity, quantum mechanics, and observations. Astrophys. Space Sci. 330, $373-$ 398, DOI 10.1007/s10509-010-0409-8

51) Smith H. From Fish to Philosopher. Little Brown, Boston, 1953.

52) Starostin EL, van der Heijden GHM. 2007. The shape of a Möbius strip. Nature Materials. 6 (8): 563-7. doi:10.1038/nmat1929

53) Storr S.J., Woolston C.M., Zhang Y., Martin S.G. Redox environment, free radical, and oxidative DNA damage. Antioxid. Redox. Signal. 2013;18:2399-2408.

54) Tegmark M. 2008. The Mathematical Universe. Foundations of Physics. February 2008, Volume 38, Issue 2, pp 101-150.

55) t'Hooft G. 1999. Quantum gravity as a dissipative deterministic system. Classical and Quantum Gravity, $16(10)$.

56) Torday JS, Sanchez-Esteban J, Rubin LP. 1998. Paracrine mediators of mechanotransduction in lung development. Am J Med Sci. 1998 Sep;316(3):205-8.

57) Torday JS, Rehan VK. 2009. Lung evolution as a cipher for physiology. Physiol Genomics. 2009 Jun 10;38(1):1-6.

58) Torday JS, Ihida-Stansbury K, Rehan VK. 2009. Leptin stimulates Xenopus lung development: evolution in a dish. Evol Dev. 2009 Mar-Apr;11(2):219-24.

59) Torday JS. 2014. On the evolution of development. Trends Dev Biol., 8:17-37.

60) Torday JS. 2015. Homeostasis as the Mechanism of Evolution. Biology (Basel). 2015 Sep 15;4(3):573-90.

61) Torday JS, Miller WB. 2016a. The Unicellular State as a Point Source in a Quantum Biological System. Biology (Basel). 2016 May 27;5(2).

62) Torday JS, Miller WB. 2016b. Phenotype as Agent for Epigenetic Inheritance. Biology (Basel). 2016 Jul $8 ; 5(3)$.

63) Torday JS, Miller WB Jr. 2017. The resolution of ambiguity as the basis for life: A cellular bridge between Western reductionism and Eastern holism. Prog Biophys Mol Biol. 2017 Dec;131:288-297.

64) Torday JS. 2018a. The Singularity of nature. Prog Biophys Mol Biol. 2018 Aug 1.

65) Torday JS. 2018b. A diachronic evolutionary biologic perspective: Reconsidering the role of the eukaryotic unicell offers a 'Timeless' biology. Prog Biophys Mol Biol. 2018 May 2.

66) Torday JS. 2018c. Pleiotropy, the physiologic basis for biologic fields. Prog Biophys Mol Biol. Aug; 136:3739.

67) Tozzi A. 2016. Borsuk-Ulam Theorem Extended to Hyperbolic Spaces. In Computational Proximity. Excursions in the Topology of Digital Images, edited by J F Peters, 169-171. doi:10.1007/978-3-319-302621.

68) Tozzi A, Peters JF. 2016b. A Topological Approach Unveils System Invariances and Broken Symmetries in the Brain. Journal of NeuroscienceResearch 94 (5): 351-65. doi:10.1002/jnr.23720.

69) Tozzi A, Peters JF. 2017a. A Symmetric Approach Elucidates Multisensory Information Integration. Information 8,1. doi: 10.3390/info8010004.

70) Tozzi A, Peters JF. 2017b. From abstract topology to real thermodynamic brain activity. Cognitive Neurodynamics. 11: 283. Doi:10.1007/s11571-017-9431-7.

71) Tozzi A, Peters JF, Fingelkurts AA, Fingelkurts AA, Marijuán PC. 2017. Topodynamics of metastable brains. Physics of Life Reviews, 21, 1-20. http://dx.doi.org/10.1016/j.plrev.2017.03.001. 
72) Tozzi A, Peters JF, Fingelkurts A, Fingelkurts A, Perlovsky L. 2018. Syntax meets semantics during brain logical computations. Progr Biophys Mol Biol. https://doi.org/10.1016/j.pbiomolbio.2018.05.010.

73) Tumulka, R. 2009. Orthonormal basis. In: D. Greenberger, K. Hentschel, F. Weinert, eds., Compendium of Quantum Physics, Springer, Berlin, https://doi-org.uml.idm.oclc.org/10.1007/978-3-540-706267135.

74) Van Raamsdonk M. 2010. Building up space-time with quantum entanglement. International Journal of Modern Physics D, 19(14): 2429-2435. https://doi.org/10.1142/S0218271810018529

75) Van Essen DC. 2005. A Population-Average, Landmark- and Surface-based (PALS) atlas of human cerebral cortex. Neuroimage. 28, 635-666.

76) Wang S, Krinks M, Lin K, Luyten FP, Moos M Jr. Frzb, a secreted protein expressed in the Spemann organizer, binds and inhibits Wnt-8. Cell. 1997 Mar 21;88(6):757-66.

77) Watanabe, T., Kan, S., Koike, T., Misaki, M., Konishi, S., Miyauchi, S. Masuda, N. 2014. Networkdependent modulation of brain activity during sleep. NeuroImage, 98, 1-10. http://doi.org/10.1016/j.neuroimage.2014.04.079.

78) Yilmaz, H. 1981. Relativity and quantum mechanics, Int. J. of Theoretical Physics 21, no. 10/11, 871-902. 